\title{
Boundary conditions and normal state for a vibrated granular fluid
}

\author{
J. Javier Brey, M. J. Ruiz-Montero, and F. Moreno \\ Física Teórica, Universidad de Sevilla, Apartado de Correos 1065, E-41080 Sevilla, Spain
}

(Received 27 March 2000)

\begin{abstract}
The steady state of a fluidized granular system confined between a vibrating wall and a reflecting one is analyzed in detail. The relationship between the velocity of the wall and the hydrodynamic profiles is established. In the limit of a large system, a peculiar normal state, independent of the details of the boundaries, is reached in the bulk. This state has a uniform pressure and a constant temperature gradient. Both quantities are not independent, but verify a closed constitutive relationship. The generality of this state and the relevant role it is expected to play in the description of vibrated granular systems is discussed.
\end{abstract}

PACS number(s): 45.70.Mg, 81.05.Rm, 51.10.+y, 05.20.Dd

\section{INTRODUCTION}

Due to kinetic-energy dissipation in collisions, energy must be continuously supplied to fluidized granular systems in order to sustain a steady state. In most of the experimental situations, this energy is given through a vibrating plate. Studying the nature of the steady state reached by the system requires both a macroscopic continuous theory describing the bulk of the system, and also an understanding of the energy exchange between the vibrating wall and the fluidized granular medium. Both aspects can be studied by means of kinetic theories of granular media, modeled after the kinetic theories of molecular gases [1-5]. Therefore, the problem is not only of evident practical interest but also very relevant from a theoretical point of view, since it provides a nontrivial test of kinetic theories.

In this paper we study a granular gas between two infinite parallel walls. One of the walls is moving and supplies energy to the system. The other one is at rest. Collisions of the particles with the walls are elastic. Besides, for the sake of simplicity, we will consider that there is not any external force such as gravity acting on the system. The motion of the wall will be modeled in such a way that a steady state is reached after some transient period of time. Several versions of this system have already been considered in the literature. In Refs. [6-8] a granular system confined in a box, where one of the walls is kept at a given temperature, is studied. These kinds of "thermal" walls are far from reality for granular systems, in which the so-called granular temperature does not have the thermodynamic meaning of the usual temperature. Moreover, the relationship between the temperature parameter of the wall and the granular temperature of the fluid next to it was not investigated. Of course, this does not invalidate a priori the conclusions reached in these works for regions of the fluid far enough from the boundaries.

A simple model leading to a relationship between the energy input by a vibrating wall and the energy dissipation in collisions was developed by Warr et al. [9], who modeled a vibrated granular medium under gravity as an isotherm atmosphere, with all particles having the mean velocity. This approach was improved by Kumaran [10] who used a Maxwellian velocity distribution. The case of absence of gravity was analyzed along the same lines in Ref. [11] where the relevance of the pressure to describe the effect of the thermal wall was emphasized. All these approaches have been discussed and compared with molecular-dynamics simulations results by McNamara and Luding [12]. Let us point out that, in spite of the relevance of the results derived in all these studies, they do not pay too much attention to the bulk properties of the system, as opposed to the "boundary layer," or to the relationship between the mechanical properties of the vibrating wall and the hydrodynamic profiles in the granular system.

Here we will present a quite detailed analysis of both the bulk of the system and the boundary conditions, and relate the parameters defining the motion of the wall with the boundary conditions to be used when solving the hydrodynamic equations to find the profiles of the hydrodynamic fields in the bulk, i.e., outside the "kinetic boundary layer." In particular, it will be shown that it is important to take into account the different mean-square velocities of particles going towards the vibrating wall and particles coming from it after colliding. Of course, the granular temperature of the gas next to the wall depends on the velocity distribution including both populations of particles.

The plan of the paper is as follows. In Sec. II, the NavierStokes-like hydrodynamic equations for a granular gas are particularized for the state under consideration and explicit expressions for the hydrodynamic profiles are given. The expressions contain, as a boundary condition, the temperature of the gas next to the vibrating wall. In the limit of a large system a linear temperature profile in the bulk is obtained as well as some scaling laws. In Sec. III, the nature of the vibrating wall is specified. For the sake of simplicity, an asymmetric wave form is used to drive the wall, so that every particle that collides with the wall finds it with the same constant velocity [9-12]. By assuming that the velocity distribution function of particles approaching the wall is Maxwellian, a set of closed equations is constructed from which the relationship between the velocity of the wall and the temperature of the gas next to it can be obtained.

The above theoretical calculations are based on the validity of the Navier-Stokes-like hydrodynamic equations to describe the granular system and on the accuracy of the approximations introduced to model the power input through the vibrating wall. The quantitative predictions of the theory are compared with direct Monte Carlo simulation results for the Boltzmann equation in Sec. IV. A good agreement is 
obtained in the bulk of the system, i.e., outside the boundary layers that appear next to the walls. In this context, it is worth mentioning that our results are also in qualitative agreement with the molecular-dynamics simulations data reported in Ref. [7]. Finally, the main conclusions are summarized and discussed in Sec. V.

\section{HYDRODYNAMIC DESCRIPTION}

The balance equations for the local number density $n(\mathbf{r}, t)$, velocity flow $\mathbf{u}(\mathbf{r}, t)$, and temperature $T(\mathbf{r}, t)$, of a fluid of smooth inelastic hard disks $(d=2)$ or spheres $(d$ $=3$ ) of mass $m$ are

$$
\begin{gathered}
\partial_{t} n+\nabla \cdot(n \mathbf{u})=0, \\
\partial_{t} \mathbf{u}+\mathbf{u} \cdot \boldsymbol{\nabla} \mathbf{u}+(m n)^{-1} \boldsymbol{\nabla} \cdot \mathbf{P}=\mathbf{0}, \\
\partial_{t} T+\mathbf{u} \cdot \boldsymbol{\nabla} T+2\left(d n k_{B}\right)^{-1}(\mathrm{P}: \boldsymbol{\nabla} \mathbf{u}+\boldsymbol{\nabla} \cdot \mathbf{q})+T \zeta=0 .
\end{gathered}
$$

These equations are derived by taking velocity moments in the pseudo-Liouville equation for the system [13]. Here $P$ is the pressure tensor, $\mathbf{q}$ is the heat flux, and $\zeta$ is a cooling rate associated to the energy dissipation in collisions. Although they can be written as functions of the $N$-particle distribution function of the system, these general expressions will not be given here. We have defined the temperature in the standard way in kinetic theory, i.e., including the Boltzmann constant $k_{B}$, although in the context of granular flows it does not have the same thermodynamic meaning as in molecular systems [14]. For the particular case of a steady state with no macroscopic velocity flow, the above equations reduce to

$$
\begin{gathered}
\boldsymbol{\nabla} \cdot \mathrm{P}=0, \\
2\left(d n k_{B}\right)^{-1} \boldsymbol{\nabla} \cdot \mathbf{q}+T \zeta=0 .
\end{gathered}
$$

Note that the above relations are direct consequence of the general balance equations (1)-(3) and, therefore, they are not restricted to low density or to the lowest orders in the gradients. For a low-density gas described by the Boltzmann equation modified to account for inelastic collisions through a constant coefficient of normal restitution $\alpha$, explicit expressions for $\mathrm{P}, \mathbf{q}$, and $\zeta$ have been obtained in the limit of small spatial inhomogeneities $[4,15]$. In the Navier-Stokes approximation, i.e., first order in the gradients of the fluxes, they are given by

$$
\begin{gathered}
\mathrm{P}=p l, \\
\mathbf{q}=-\kappa \boldsymbol{\nabla} T-\mu \boldsymbol{\nabla} n, \\
\zeta=\zeta^{(0)}+\zeta^{(2)} .
\end{gathered}
$$

In Eq. (6), $p=n k_{B} T$ is the pressure and I the unit tensor. The term $\zeta^{(0)}$ in Eq. (8) is of zeroth order in the gradients of the fields, while $\zeta^{(2)}$ contains the second order in the gradients contributions to the cooling rate. These contributions are expected to be very small as compared with the heat flow and the pressure tensor [16] and, therefore, they will be neglected in the following. The heat conductivity $\kappa$ and the transport coefficient $\mu$ have the form

$$
\kappa=\kappa^{*}(\alpha) \kappa_{0}(T), \quad \mu=\mu^{*}(\alpha) \frac{T \kappa_{0}(T)}{n},
$$

while it is

$$
\zeta^{(0)}=\frac{p}{\eta_{0}(T)} \zeta^{*}(\alpha)
$$

where $\kappa_{0}$ and $\eta_{0}$ are the elastic heat conductivity and shear viscosity, respectively, and $\kappa^{*}(\alpha), \nu^{*}(\alpha)$, and $\zeta^{*}(\alpha)$ are dimensionless functions of the coefficient of normal restitution $\alpha$. The explicit expressions of all these quantities are given in the Appendix.

The specific problem we are interested in is a system enclosed between two infinite parallel walls located at $x=0$ and $x=L$, respectively. From symmetry considerations, gradients of the hydrodynamic fields are expected only in the $x$ direction and Eqs. (4) and (5) become

$$
\begin{gathered}
\frac{\partial}{\partial x} p=0 \\
2\left(d n k_{B}\right)^{-1}\left[\kappa^{*}(\alpha)-\mu^{*}(\alpha)\right] \frac{\partial}{\partial x}\left(\kappa_{0} \frac{\partial T}{\partial x}\right)-\frac{\zeta^{*} p T}{\eta_{0}}=0 .
\end{gathered}
$$

It is now convenient to introduce a dimensionless space variable $l$ by

$$
d l=\frac{d x}{\lambda(x)}
$$

where $\lambda(x)$ is the local mean-free-path for hard disks or spheres,

$$
\lambda(x)=\left[C \sigma^{d-1} n(x)\right]^{-1} .
$$

Here $C$ is a constant depending on the dimension of the system, namely, $C=2 \sqrt{2}$ for $d=2$ and $C=\pi \sqrt{2}$ for $d=3$. In terms of the new variable, Eq. (12) reads

$$
\frac{\partial^{2}}{\partial l^{2}} T(l)^{1 / 2}=a(\alpha) T(l)^{1 / 2}
$$

with

$$
a(\alpha)=\frac{32(d-1) \pi^{d-1} \zeta^{*}(\alpha)}{(d+2)^{3} C^{2} \Gamma(d / 2)^{2}\left[\kappa^{*}(\alpha)-\mu^{*}(\alpha)\right]} .
$$

The above expressions clearly indicate the intrinsic connection between gradients and dissipation in the system. In mean-free-path units the former are determined by $\sqrt{a(\alpha)}$. Therefore, application of the Navier-Stokes equations, which assume small gradients, to the present situation, is limited to the low-inelasticity limit [7]. The general solution of Eq. (15) is

$$
T(l)^{1 / 2}=A e^{-\sqrt{a(\alpha)} l}+B e^{\sqrt{a(\alpha)} l}
$$

with $A$ and $B$ arbitrary factors to be determined from the boundary conditions. We are going to consider that there is a vibrating wall at $x=0$ supplying energy to the system and a 


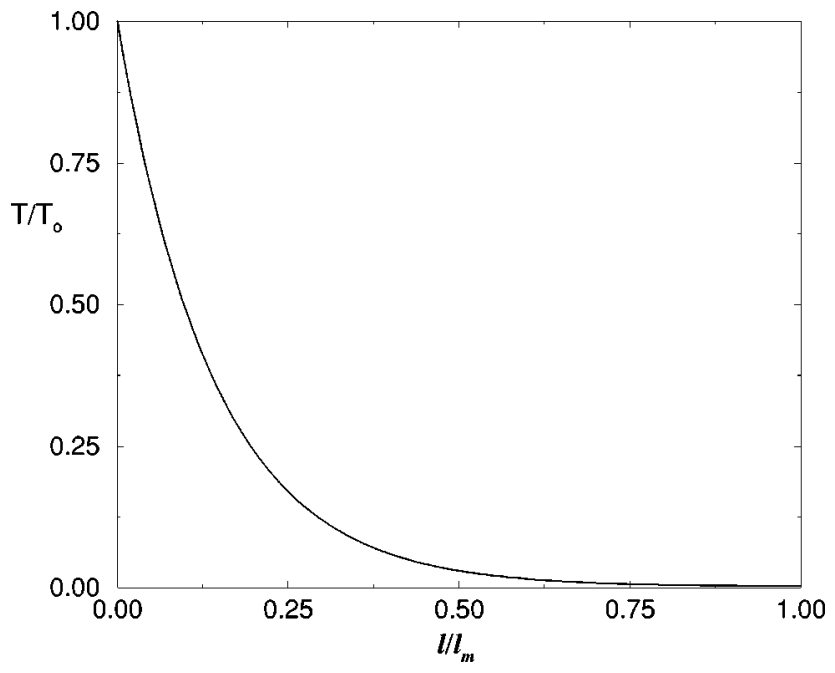

FIG. 1. Plot of Eq. (19) for a two-dimensional system with $\alpha$ $=0.99$ and $l_{m}=80$.

reflecting wall at $x=L$. Denoting by $T_{0}$ the temperature of the gas next to the vibrating wall, we can express the above boundary conditions by

$$
T(l=0)=T_{0}, \quad\left(\frac{\partial T}{\partial l}\right)_{l=l_{m}}=\left(\frac{\partial T}{\partial x}\right)_{x=L}=0 .
$$

We have taken the origin for the $l$ coordinate at $x=0$, and $l_{m}$ is the value of $l$ for $x=L$. Of course, there still remains the problem of relating $T_{0}$ with the parameters characterizing the motion of the vibrating wall. This will be discussed in Sec. III. When Eqs. (18) are used to fix the values of $A$ and $B$ in Eq. (17), it takes the form

$$
T(l)=T_{0}\left\{\frac{\cosh \left[\sqrt{a(\alpha)}\left(l_{m}-l\right)\right]}{\cosh \left[\sqrt{a(\alpha)} l_{m}\right]}\right\}^{2} .
$$

For illustration, in Fig. 1, we plot this expression for given values of $\alpha$ and $l_{m}$.

Let $N_{x}$ be the number of particles in the system per unit of section perpendicular to the $x$ axis. It is

$$
N_{x}=\int d x n(x)=\frac{l_{m}}{C \sigma^{d-1}} .
$$

We can also define an average transversal density $\bar{n}_{x}$ $=N_{x} / L$. From Eq. (20) it follows that $l_{m}$ grows linearly with the linear size $L$ of the system if the value of $\bar{n}_{x}$ is kept constant.

Use of Eq. (19) into Eq. (13) provides an explicit relationship between the $x$ and $l$ length scales,

$$
\begin{aligned}
x= & \frac{k_{B} T_{0}}{4 C \sigma^{d-1} p \sqrt{a(\alpha)}\left\{\cosh \left[\sqrt{a(\alpha)} l_{m}\right]\right\}^{2}} \\
& \times\left\{2 \sqrt{a(\alpha)} l+\sinh \left[2 \sqrt{a(\alpha)} l_{m}\right]\right. \\
& \left.-\sinh \left[2 \sqrt{a(\alpha)}\left(l_{m}-l\right)\right]\right\} .
\end{aligned}
$$

The temperature at the reflecting wall, i.e., for $x=L$ or $l$ $=l_{m}$ is

$$
T_{m}=T_{0}\left\{\frac{1}{\cosh \left[\sqrt{a(\alpha)} l_{m}\right]}\right\}^{2}
$$

and particularization of Eq. (21) for $x=L$ gives

$$
L=\frac{k_{B} T_{m}}{4 C \sigma^{d-1} p \sqrt{a(\alpha)}}\left\{2 \sqrt{a(\alpha)} l_{m}+\sinh \left[2 \sqrt{a(\alpha)} l_{m}\right]\right\}
$$

Up to this point all the results we have derived follow directly from the Navier-Stokes-like hydrodynamic equations. No additional approximation has been introduced. Let us now assume that $L$ is very large so that $\sqrt{a(\alpha)} l_{m} \gg 1$. Because of Eq. (22), this is equivalent to $T_{m} \ll T_{0}$. In fact, in this limit Eqs. (22) and (23) can be approximated by

$$
\begin{gathered}
T_{m} \simeq 4 T_{0} e^{-2 \sqrt{a(\alpha)} l_{m}}, \\
p \simeq \frac{k_{B} T_{0}}{2 C \sqrt{a(\alpha)} \sigma^{d-1} L} .
\end{gathered}
$$

The latter is quite a surprising result since it indicates that the pressure in the system, in the region in which our approximation is valid, does not depend on the total number of particles it contains, but it is determined by the "temperature gradient" $T_{0} / L$. We will return to the discussion of the above relationship in Sec. V. If, in addition to considering a large system, we restrict ourselves to distances from the vibrating wall such that $l \ll l_{m}$, it is seen that the temperature decays exponentially with $l$ in that region,

$$
T(l) \simeq T_{0} e^{-2 \sqrt{a(\alpha)} l},
$$

and from Eq. (21) it follows that the temperature profile is linear in the actual space variable $x$,

$$
T(x) \simeq T_{0}\left(1-\frac{x}{L}\right) .
$$

It is worth to emphasize that Eqs. (26) and (27), being restricted to the limit $x \ll L$, cannot be applied close to the reflecting wall. In fact, in that limit Eq. (26) leads to a value of the temperature at the reflecting wall that differs by a factor of 4 from the exact value given by Eq. (24). On the other hand, in the region in which both equations apply, it is

$$
n(x)=\frac{1}{2 C \sqrt{a(\alpha)} \sigma^{d-1}(L-x)},
$$

i.e., the density profile is only a function of the size $L$ of the system and the coefficient of restitution $\alpha$, but it depends neither on the properties of the vibrating wall, which determine $T_{0}$, nor on the number of particles in the system. The linear behavior in Eq. (27) was already noted by Grossman et al. [7] who also observed it in molecular-dynamics simulations. 


\section{BOUNDARY CONDITIONS}

In order to close the description obtained in the previous section, we still need an expression for the temperature $T_{0}$ of the gas next to the vibrating wall in terms of the parameters defining the motion of the latter. For the sake of simplicity, we will consider that the wall moves in a sawtooth manner, i.e., all particles colliding with the wall find it with a constant velocity $v_{b}$ in the direction of positive $x[11,12]$. Moreover, the amplitude of the wall motion is very small as compared with the mean-free-path of the gas in the vicinity of the wall. As a consequence, the position of the wall can be taken as fixed at $x=0$ in a good approximation. In this way, we avoid the presence of heat pulses propagating from the wall [11].

We treat the wall-particle collisions as elastic, so that when a particle of velocity $\mathbf{v}$, with $v_{x}<0$, collides with the wall, it changes its velocity into $\mathbf{v}^{\prime}$ given by

$$
v_{x}^{\prime}=2 v_{b}-v_{x}, \quad \mathbf{v}_{\perp}^{\prime}=\mathbf{v}_{\perp}
$$

Here $\mathbf{v}_{\perp}$ denotes the vector component of the velocity perpendicular to the $x$ axis. The energy gained by the particle in the collision is

$$
\Delta E=2 m\left(v_{b}^{2}-v_{b} v_{x}\right)
$$

The rate of energy input through the vibrating wall per unit of length (for $d=2$ ) or area (for $d=3$ ) can be expressed as

$$
Q=\int d \mathbf{v} \theta\left(-v_{x}\right)\left|v_{x}\right| f_{0}(\mathbf{v}) \Delta E
$$

where $\theta$ is the Heaviside step function and $f_{0}(\mathbf{v})$ the oneparticle distribution function of the gas next to the wall. It is important to realize that to compute $Q$ only the precollisional distribution $\theta\left(-v_{x}\right) f_{0}(\mathbf{v})$ for particles approaching the wall is needed. In fact, if this precollisonal distribution is known, the collisional rule in Eqs. (29) determines the postcollisional distribution as a functional of it. Let us write

$$
f_{0}(\mathbf{v})=f_{0}^{(-)}(\mathbf{v}) \theta\left(-v_{x}\right)+f_{0}^{(+)}(\mathbf{v}) \theta\left(v_{x}\right)
$$

Then the boundary condition implied by the vibrating wall can be expressed as

$f_{0}^{(+)}(\mathbf{v}) \theta\left(v_{x}\right) v_{x}=f_{0}^{(-)}\left(2 v_{b}-v_{x}, \mathbf{v}_{\perp}\right) \theta\left(v_{x}-2 v_{b}\right)\left(v_{x}-2 v_{b}\right)$.

It is easily verified that the above relationship guarantees that the local velocity flow vanishes at the wall as it should. For the number of particles density one gets

$$
n_{0}=2 n_{0}^{(-)}+2 v_{b} \int d \mathbf{v} \frac{1}{v_{x}-2 v_{b}} f_{0}^{(-)}(\mathbf{v}) \theta\left(-v_{x}\right)
$$

with

$$
n_{0}^{(-)}=\int d \mathbf{v} f_{0}^{(-)}(\mathbf{v}) \theta\left(-v_{x}\right)
$$

Similarly, for the temperature it is found that

$$
\begin{aligned}
& \frac{d}{2} n_{0} k_{B} T_{0}=d n_{0}^{(-)} k_{B} T_{0}^{(-)}-m v_{b} n_{0}^{(-)} u_{0, x}^{(-)} \\
& +v_{b} \int d \mathbf{v} \frac{m v_{\perp}^{2}}{v_{x}-2 v_{b}} f_{0}^{(-)} \theta\left(-v_{x}\right), \\
& n_{0}^{(-)} u_{0, x}^{(-)}=\int d \mathbf{v} v_{x} f_{0}^{(-)}(\mathbf{v}) \theta\left(-v_{x}\right), \\
& \frac{d}{2} n_{0}^{(-)} k_{B} T_{0}^{(-)}=\int d \mathbf{v} \frac{1}{2} m v^{2} f_{0}^{(-)}(\mathbf{v}) \theta\left(-v_{x}\right) .
\end{aligned}
$$

To proceed further we need the expression of $f_{0}^{(-)}(\mathbf{v}) \theta\left(-v_{x}\right)$. A simple choice, already used by previous authors $[10,17]$, is to assume that it factorizes and is Gaussian for all the components, i.e., we assume that

$$
\begin{aligned}
f_{0}^{(-)}(\mathbf{v}) \theta\left(-v_{x}\right)= & 2 n_{0}^{(-)} \varphi_{M B}\left(\mathbf{v}_{\perp}\right) \\
& \times\left(\frac{m}{2 \pi k_{B} T_{1}}\right)^{1 / 2} e^{-\left(m v_{x}^{2} / 2 k_{B} T_{1}\right)} \theta\left(-v_{x}\right), \\
\varphi_{M B}\left(\mathbf{v}_{\perp}\right)= & \left(\frac{m}{2 \pi k_{B} T_{0}}\right)^{(d-1) / 2} e^{-m v_{\perp}^{2} / 2 k_{B} T_{0}}
\end{aligned}
$$

This factorization is expected to give a good approximation to the actual distribution of the fluid, although a careful discussion of this point can only be carried out in the context of a kinetic equation, what is beyond the scope of this paper. The presence of $n_{0}^{(-)}$and $T_{1}$ in Eq. (39) is required by consistency with the results derived in the previous section. The distinction between $2 n_{0}^{(-)}$and $n_{0}$ on the one hand, and between $T_{1}$ and $T_{0}$ on the other, is a main point in the theory we present here. The distribution of the velocity parallel to the wall is also Maxwellian with the actual local temperature $T_{0}$. It must be noticed that $T_{0}^{(-)}$, as defined in Eq. (38), differs from the parameter $T_{1}$ introduced in Eq. (39).

The form of Eq. (39) deserves an additional comment. In principle, there is no reason to expect that the marginal velocity distribution for $v_{x}$ exhibits a maximum at $v_{x}=0$. In fact, numerical solutions of the Boltzmann equation to be discussed in the next section show that the maximum is located at a negative velocity of the order of a few times $v_{b}$. This is clearly a nonhydrodynamic boundary effect and we will neglect it in our approximation, not only for simplicity reasons but also because we believe it is consistent with the extrapolation of the linear temperature profile to $x=0$ predicted by the hydrodynamic description [see Eq. (27)]. More will be said about this point in the next section.

When the distribution function in Eq. (39) is used, Eqs. (34) and (36) become

$$
\begin{gathered}
\rho=1-2 \pi^{-1 / 2} \tilde{v}_{b} \int_{0}^{\infty} d v \frac{e^{-v^{2}}}{v+2 \tilde{v}_{b}}, \\
\rho \vartheta=1+\frac{2 \tilde{v}_{b}}{\pi^{1 / 2}},
\end{gathered}
$$


respectively, where

$$
\tilde{v}_{b}=\left(\frac{m}{2 k_{B} T_{1}}\right)^{1 / 2} v_{b}
$$

and

$$
\rho=\frac{n_{0}}{2 n_{0}^{(-)}}, \quad \vartheta=\frac{T_{0}}{T_{1}} .
$$

In the same Gaussian approximation, Eq. (31) takes the form

$$
Q=2 m n_{0}^{(-)}\left[\frac{k_{B} T_{1}}{m} v_{b}+\left(\frac{2 k_{B} T_{1}}{\pi m}\right)^{1 / 2} v_{b}^{2}\right] .
$$

To close the problem we need an alternative expression for $Q$ as a function of the hydrodynamic variables in the system. This follows, for instance, from Eq. (7) in the limit $x \rightarrow 0^{+}$,

$$
Q=-\left[\kappa^{*}(\alpha)-\mu^{*}(\alpha)\right] \kappa_{0}\left(T_{0}\right)\left(\frac{\partial T}{\partial x}\right)_{x \rightarrow 0^{+}},
$$

valid in the limit of large size L. Equating Eqs. (45) and (46) and use of Eqs. (25) and (27) yields

$$
\tilde{v}_{b}+2 \pi^{-1 / 2} \widetilde{v}_{b}^{2}=b(\alpha) \vartheta^{3 / 2} \rho,
$$

where

$$
\begin{aligned}
b(\alpha)= & \frac{d(d+2)^{2} 2^{1 / 2}}{16(d-1)} \pi^{-(d-1) / 2} \Gamma\left(\frac{d}{2}\right) C \sqrt{a(\alpha)} \\
& \times\left[\kappa^{*}(\alpha)-\mu^{*}(\alpha)\right] .
\end{aligned}
$$

Equations (41), (42), and (48) give the values of $\tilde{v}_{b}, \rho$, and $\vartheta$, for a given coefficient of restitution $\alpha$. No other parameter of the system is involved. Then, from these values and the velocity of the vibrating wall $v_{b}$, the temperatures $T_{1}$ and $T_{0}$ are obtained. This fully specifies the hydrodynamic profiles of the system, as discussed in Sec. II. A direct consequence of the form of the above equations is that the temperature $T_{0}$ of the gas next to the vibrating wall, scales with $v_{b}^{2}$, being independent of the average density and the size $L$ of the system. Moreover, from Eq. (25) it follows that also the pressure $p$ is proportional to $v_{b}^{2}$. These scaling properties will be checked in the numerical simulations of the Boltzmann equation to be discussed in the next section.

\section{MONTE CARLO SIMULATIONS}

To check the accuracy of the hydrodynamic predictions we have derived in the previous sections, we have compared them with numerical solutions of the Boltzmann equation constructed by means of the direct simulation Monte Carlo (DSMC) method [18]. The general idea in which this method is based is to generate a Markov process that mimics the dynamical processes described by the Boltzmann equation. Although the method was originally devised for elastic particles, inelasticity in collisions is incorporated just by changing the expressions of the postcollisional velocities as compared with the elastic case [19]. We will not repeat here the technical details of the DSMC method that can be found in

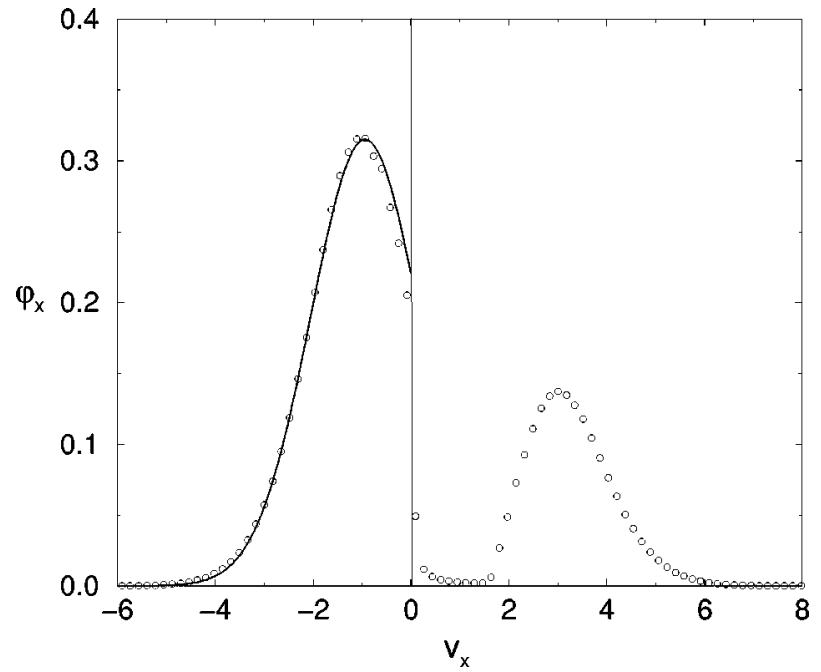

FIG. 2. Marginal distribution function $\varphi_{x}$ of the $x$ component of the velocity in a layer of width 0.25 next to a vibrating wall, moving with velocity $v_{b}=0.8$. The length of the system is $L=60$ and the coefficient of restitution is $\alpha=0.975$. All the quantities are measured in the units defined in the text. The points are from the simulation and the continuous line is a fit to a Gaussian distribution of the data corresponding to $v_{x}<0$.

Ref. [18]. In order to value the relevance of the results of the comparison, it must be kept in mind that no hydrodynamic concepts are introduced externally when numerically solving the Boltzmann equation. Also the walls are treated in a purely mechanical way and the size of the boundary layers is not known a priori.

We have simulated a system of hard disks $(d=2)$ with several values of the coefficient of restitution $\alpha$ in the interval $[0.9,0.99]$. We restrict ourselves to this interval since the Navier-Stokes equations are not expected to hold for larger inelasticity, as discussed below Eq. (16). For each value of $\alpha$, we have varied the size $L$ of the system and the velocity $v_{b}$ of the vibrating wall in order to test the scaling laws derived in the previous sections. All the results we will present in the following correspond to the steady state reached by the system after a transient time that depends on parameters of the system under consideration. We use the particle mass $m$ to define the unit of mass, and the homogeneous mean-free-path $\lambda_{H}=L /\left(2 \sqrt{2} N_{x} \sigma\right)$ as the unit of distance. Finally, the unit of temperature is defined by the average kinetic energy of the initial state.

The theoretical discussion of the boundary conditions we have carried out is based on the assumption that the precollisional marginal velocity distribution next to the vibrating wall is Gaussian and centered at the origin. Then, the postcollisonal velocity distribution is given by Eq. (33). In Fig. 2 we present the marginal distribution function of the $x$ component of the velocity,

$$
\varphi_{x}\left(v_{x}\right)=\frac{1}{n_{0}} \int d v_{y} f_{0}(\mathbf{v})
$$

next to the wall for $\alpha=0.975$ and $L=60$. The plotted distribution function corresponds to the layer $0<x<0.25$ and the velocity of the wall is $v_{b}=0.8$. It is observed that the numerical data for $v_{x}<0$ (in-flowing particles) are well fitted 


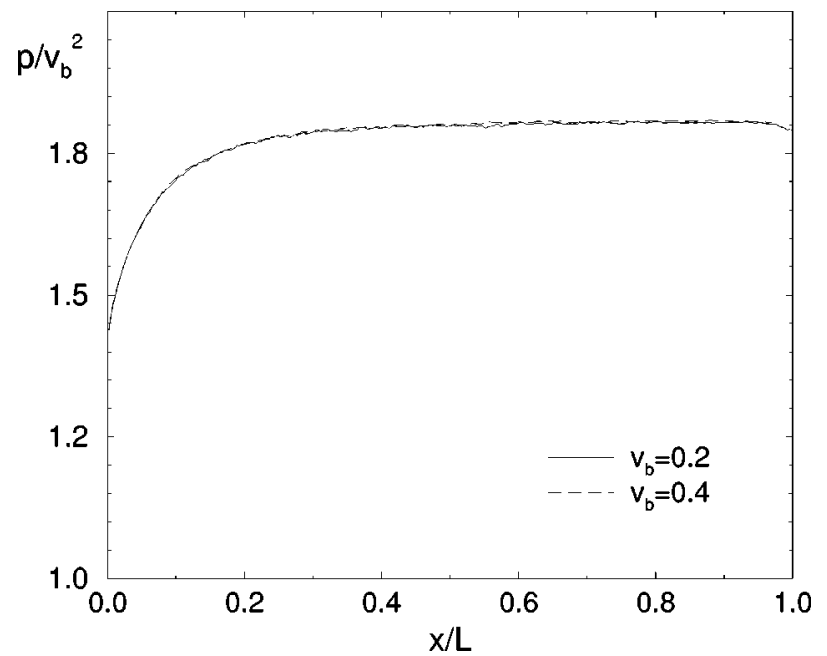

FIG. 3. Simulation results for the pressure profile scaled with the square of the velocity of the vibrating wall $v_{b}$ for a system with $L=80$ and $\alpha=0.99$. Two different values of $v_{b}$ have been considered. The quantities are measured in the reduced units defined in the text.

by a Gaussian. Also, we have verified that the distribution for $v_{x}>0$ (out-flowing particles) is accurately generated by Eq. (33). Nevertheless, the maximum of the distribution for negative velocities is clearly displaced from the origin as already mentioned in the previous section. This displacement tends to vanish when the distance from the wall increases.

As a first test of the hydrodynamic predictions, we plot in Fig. 3 the pressure profile for a system with $L=80$ and $\alpha$ $=0.99$. Two different values of velocity of the wall have been considered to show the scaling of the pressure with $v_{b}^{2}$. It is seen that the pressure is really constant in most of the system, although the presence of a boundary layer is clearly identified. This boundary layer cannot be explained by the Navier-Stokes equations and when comparing the numerical results with the theory predictions we will consider the value of the pressure in the bulk, that in Fig. 3, corresponds roughly to the interval $0.25<x / L<0.95$. Because of the shape of the pressure in the boundary layer, we have preferred to consider the energy flux as a function of the temperature profile instead of the pressure in Sec. III, since the latter quantity is hard to extrapolate next to the vibrating wall.

In Fig. 4 we present the temperature profile obtained for $\alpha=0.95$ and several values of $v_{b}$ and $L$. It is found that $T / v_{b}^{2}$ is a function of $x / L$ for given $\alpha$ as predicted by the theory. Also, the temperature profile is quite linear outside the boundary layer next to the vibrating wall. Similar results have been obtained for other values of $\alpha$ in the interval studied. In fact, the numerical data show that the width of the boundary layer becomes narrower as $\alpha$ decreases. The theoretical prediction for the temperature profile is given by Eq. (27) with $T_{0}$ determined by the solution of Eqs. (41), (42), and (48), as discussed at the end of the previous section. Then, we have fitted the numerical data for the temperature profile in the linear region to a function of the same form as Eq. (27) with only one adjustable parameter corresponding to the temperature at the wall $T_{0}$. In this respect, it seems sensible to think that neglecting the displacement of the maxi-

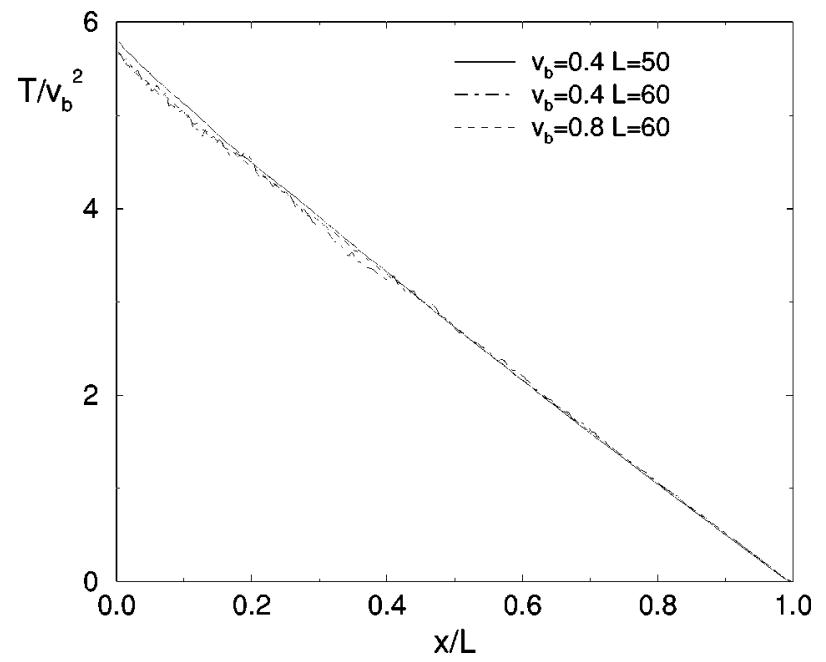

FIG. 4. Simulation results for the temperature profile scaled with $v_{b}^{2}$ for a system with $\alpha=0.95$. Different values of $L$ and $v_{b}$ have been used, as indicated in the figure.

mum of the precollisional velocity distribution next to the wall, as made in Sec. III, is consistent with the extrapolation of the linear bulk behavior up to the vibrating wall, since the gap in the velocity distribution disappears in the bulk.

The comparison of theory and simulation for the temperature $T_{0}$ is presented in Fig. 5. Each of the points has been obtained by averaging the numerical results for several values of $L$ and $v_{b}$. In any case, the dispersion of these values was always smaller than $5 \%$. The agreement between theory and simulation is surprisingly good. To underline the importance of differentiating between the precollisonal velocity distribution next to the wall and the postcollisonal one, i.e., between $T_{0}^{(-)}$and $T^{(0)}$, we have also plotted the prediction obtained if a single Gaussian distribution is used to describe the velocity distribution in that region. This is equivalent to put $\theta=\rho=1$ in Eq. (47). From the figure it follows that this latter approximation introduces a significant deviation in the prediction for $T_{0}$.

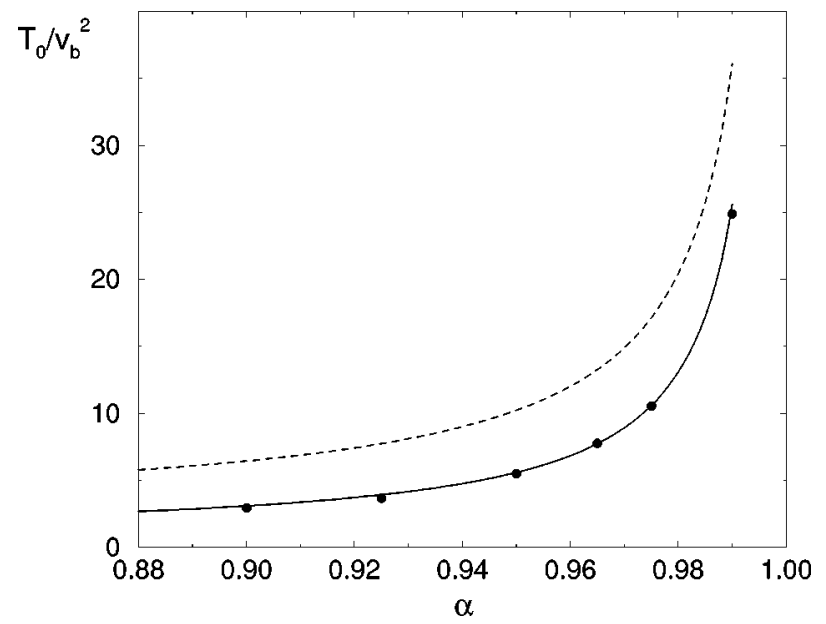

FIG. 5. Temperature $T_{0}$ of the granular gas next to vibrating wall scaled with $v_{b}^{2}$ as a function of the coefficient of restitution $\alpha$. The points are from the simulations, the continuous line is the theoretical prediction derived here, and the dashed line the result obtained using a single Gaussian for the velocity distribution next to the wall. 


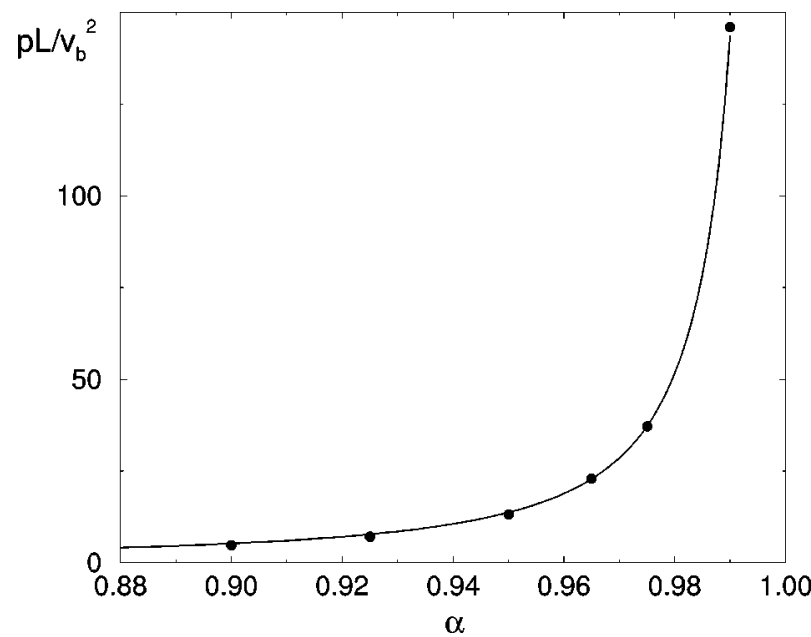

FIG. 6. Scaled pressure in the bulk as a function of the coefficient of restitution $\alpha$. The points are from the Monte Carlo simulations and the continuous line from the prediction by the theory.

The values of the pressure, outside the boundary layer, as a function of the coefficient of restitution, are plotted in Fig. 6. Again the agreement between the theoretical predictions and the Monte Carlo simulations is quite good, the relative differences being always smaller than $1 \%$. In the simulations we have noted that the boundary layer in the pressure is rather broader than the one in the temperature.

\section{DISCUSSION}

The steady state of a granular gas confined between a vibrating wall and a purely reflecting one has been examined by means of the hydrodynamic equations. In the limit of a large system and in regions located far from the boundaries, the state of the system becomes particularly simple. The pressure is uniform and the temperature profile is linear. An important point is that both quantities, pressure and gradient of temperature, are not independent, but are related through the parameters of the system. This can be understood as follows. Let us look for a solution to the hydrodynamic equations describing a state with constant pressure $p$ and a temperature profile of the form

$$
T(x)=A x, \quad x \geqslant 0, A>0 .
$$

The question is whether such a solution exists. Substitution of Eq. (50) into Eq. (12) shows that the condition for it is that the pressure has the value

$$
p=\frac{k_{B} A}{2 C \sigma^{d-1} \sqrt{a(\alpha)}}
$$

that is equivalent to Eq. (25). Therefore, Eqs. (50) and (51) define an exact solution of the Navier-Stokes-like equations for a granular gas. It is precisely this state, the one which is observed in the simulations in the bulk of a system that is being supplied energy through a vibrating wall. The state is peculiar in the sense that pressure and temperature gradient cannot be fixed arbitrarily, but they are related by Eq. (51). The relevance of this state is reinforced by the fact that it also corresponds to exact solutions, without approximations, of the Boltzmann equation for inelastic hard spheres or disks. It plays the role of a reference state for the study of vibrated granular systems. These points will be discussed elsewhere.

The above discussion suggests a qualitative explanation of the origin of the boundary layer next to the reflecting wall observed in the simulations. Given the temperature of the gas in the vicinity of the vibrating wall, that is determined by the velocity of the wall as analyzed in Sec. III, the system tries to establish a constant-temperature gradient with a uniform pressure given by Eq. (51). Both profiles determine in turn the density profile, through the equation of state. Nevertheless, such a state cannot be reached in the complete system because, in general, the imposed total number of particles does not fit with the density profile the system is trying to establish. As a compromise, the system tends to the state defined by Eqs. (50) and (51) in a region starting next to the vibrating wall and concentrates all the surplus particles in the opposite region, in a kind of "condensated region." Of course, the nature of this region cannot be analyzed by means of the Boltzmann equation that neglects the size of the particles. This picture is also consistent with the experimental findings as well as with the molecular-dynamics simulations.

In this paper we have also analyzed the relationship between the velocity of the vibrating wall and the hydrodynamic profiles in the system. We have shown the importance of taking into account the anisotropy of the velocity distribution function next to the wall, that is a direct consequence of the rules defining the collisions of a particle against a moving wall.

\section{ACKNOWLEDGMENTS}

This research was partially supported by the Dirección General de Investigación Científica y Técnica (Spain) through Grant No. PB98-1124.

\section{APPENDIX}

In this appendix, the explicit expressions of the several coefficients introduced in Eqs. (9) and (10) are given. The elastic heat conductivity and shear viscosity are

$$
\kappa_{0}=\frac{d(d+2)^{2}}{16(d-1)} \Gamma\left(\frac{d}{2}\right) \pi^{-(d-1) / 2} k_{B}\left(\frac{k_{B} T}{m}\right)^{1 / 2} \sigma^{-(d-1)}
$$

and

$$
\eta_{0}=\frac{2+d}{8} \Gamma\left(\frac{d}{2}\right) \pi^{-(d-1) / 2}\left(m k_{B} T\right)^{1 / 2} \sigma^{-(d-1)},
$$

respectively. The dimensionless quantities are functions of the coefficient of restitution given by

$$
\kappa^{*}(\alpha)=\left[\nu^{*}(\alpha)-\frac{2 d}{d-1} \zeta^{*}(\alpha)\right]^{-1}\left[1+c^{*}(\alpha)\right]
$$




$$
\begin{aligned}
\mu^{*}(\alpha)= & 2 \zeta^{*}(\alpha)\left[\kappa^{*}(\alpha)+\frac{(d-1) c^{*}(\alpha)}{2 d \zeta^{*}(\alpha)}\right] \\
& \times\left[\frac{2(d-1)}{d} \nu^{*}(\alpha)-3 \zeta^{*}(\alpha)\right]^{-1}, \\
\zeta^{*}(\alpha) & =\frac{2+d}{4 d}\left(1-\alpha^{2}\right)\left[1+\frac{3 c^{*}(\alpha)}{32}\right] .
\end{aligned}
$$

In the above expressions,

$$
\begin{gathered}
\nu^{*}=\frac{1+\alpha}{d-1}\left[\frac{d-1}{2}+\frac{3(d+8)(1-\alpha)}{16}\right. \\
\left.+\frac{4+5 d-3(4-d) \alpha}{1024} c^{*}(\alpha)\right] \\
c^{*}=\frac{32(1-\alpha)\left(1-2 \alpha^{2}\right)}{9+24 d+(8 d-41) \alpha+30 \alpha^{2}(1-\alpha)} .
\end{gathered}
$$

[1] C.K.W. Lun, S.B. Savage, D.J. Jeffrey, and N. Chepurniy, J. Fluid Mech. 140, 223 (1984).

[2] J.T. Jenkins and M.W. Richman, Arch. Ration. Mech. Anal. 87, 355 (1985); Phys. Fluids 28, 3485 (1986).

[3] A. Goldshtein and M. Shapiro, J. Fluid Mech. 282, 75 (1995).

[4] J.J. Brey, J.W. Dufty, C.S. Kim, and A. Santos, Phys. Rev. E 58, 4638 (1998).

[5] V. Garzó and J.W. Dufty, Phys. Rev. E 59, 5895 (1999).

[6] Y. Du, H. Li, and L.P. Kadanoff, Phys. Rev. Lett. 74, 1268 (1995).

[7] E.L. Grossman, T. Zhou, and E. Ben-Naim, Phys. Rev. E 55, 4200 (1997).

[8] J.J. Brey and D. Cubero, Phys. Rev. E 98, 57 (1998).

[9] S. Warr, J.M. Huntley, and G.T.H. Jacques, Phys. Rev. E 52, 5583 (1995).

[10] V. Kumaran, Phys. Rev. E 57, 5660 (1998); P. Sunthar and V. Kumaran, ibid. 60, 1951 (1999).
[11] S. McNamara and J-L. Barrat, Phys. Rev. E 55, 7767 (1997).

[12] S. McNamara and S. Luding, Phys. Rev. E 58, 813 (1998).

[13] J.J. Brey, J.W. Dufty, and A. Santos, J. Stat. Phys. 87, 1051 (1997); T.C.P. Van Noije, M.H. Ernst, and R. Brito, Physica A 251, 266 (1998).

[14] C.S. Campbell, Annu. Rev. Fluid Mech. 22, 57 (1990).

[15] J.J. Brey and D. Cubero, in Granular Gases, edited by S. Luding and T. Pöschel, Lecture Notes in Physics (Springer Verlag, Berlin, 2000).

[16] This has been explicitely verified for the linear part of $\zeta^{(2)}$ in Ref. [4].

[17] J. Eggers, Phys. Rev. Lett. 83, 5322 (1999).

[18] G. Bird, Molecular Gas Dynamics and the Direct Simulation of Gas Flows (Clarendon Press, Oxford, 1994).

[19] J.J. Brey, M.J. Ruiz-Montero, and D. Cubero, Phys. Rev. E 54, 3664 (1996); J.J. Brey, D. Cubero, and M.J. Ruiz-Montero, ibid. 59, 1256 (1999). 